\title{
CONVOLUTIONS OF UNIVALENT FUNCTIONS WITH POSITIVE COEFFICIENTS
}

\author{
B. A. URALEGADDI AND A. R. DESAI
}

\begin{abstract}
A.bstract. Let $f(z)=z+\sum_{n=2}^{\infty} a_{n} z^{n}, a_{n} \geq 0$ and $g(z)=z+\sum_{n=2}^{\infty} b_{n} z^{n}, b_{n} \geq 0$. We investigate some properties of $h(z)=f(z) * g(z)=z+\sum_{n=2}^{\infty} a_{n} b_{n} z^{n}$ where $f(z)$ and $g(z)$ belongs to certain subclasses of starlike and convex functions.
\end{abstract}

\section{Introduction}

Let $S$ be the class consisting of the functions of the form $f(z)=z+\sum_{n=2}^{\infty} a_{n} z^{n}$ that are analytic and univalent in the unit disk $E=\{z:|z|<1\}$. A function $f \in S$ is said to be starlike of order $\alpha, 0 \leq \alpha<1$, if $\operatorname{Re}\left\{\frac{z f^{\prime}(z)}{f(z)}\right\}>\alpha$ for $z \in E$ and it said to be convex of order $\alpha, 0 \leq \alpha<1$, if $\operatorname{Re}\left\{1+\frac{z f^{\prime \prime}(z)}{f^{\prime}(z)}\right\}>\alpha$ for $z \in E$. These classes are respectively denoted by $S^{*}(\alpha)$ and $K(\alpha) . S^{*}(0)=S^{*}$ and $K(0)=K$ are respectively the classes of starlike and convex functions in $S$.

Let $f(z)=z+\sum_{n=2}^{\infty} a_{n} z^{n}$ and $g(z)=z+\sum_{n=2}^{\infty} b_{n} z^{n}$. Then the Hadmard product (convolution) $(f * g)(z)$ of functions $f(z)$ and $g(z)$ is defined by $(f * g)(z)=z+$ $\sum_{n=2}^{\infty} a_{n} b_{n} z^{n}$.

Recetly Ruscheweyh and Sheil-Small [1] proved the Polya-Schoenberg conjecture that if $f(z)=z+\sum_{n=2}^{\infty} a_{n} z^{n} \in K$ and $g(z)=z+\sum_{n=2}^{\infty} b_{n} z^{n} \in K$ then $h(z)=f(z) * g(z)=$ $z+\sum_{n=2}^{\infty} a_{n} b_{n} z^{n} \in K$. Further in [2] Shild and Silverman considered convolutions of univalent functions with negative coefficients.

For $1<\beta \leq \frac{4}{3}$ and $z \in E$ let $M(\beta)=\left\{f \in S: \operatorname{Re} \frac{z f^{\prime}(z)}{f(z)}<\beta\right\}$ and $L(\beta)=\{f \in$ $\left.S: \operatorname{Re}\left(1+\frac{z f^{\prime \prime}(z)}{f^{\prime}(z)}\right)<\beta\right\}$. Let $V$ be the subclass of $S$ consisting of functions of the form $f(z)=z+\sum_{n=2}^{\infty} a_{n} z^{n}, a_{n} \geq 0$. Let $V^{*}(\alpha)=S^{*}(\alpha) \cap V, V_{k}(\alpha)=K(\alpha) \cap V$ and $V(\beta)=M(\beta) \cap V, U(\beta)=L(\beta) \cap V . V^{*}(0)=V^{*}$ and $V_{k}(0)=V_{k}$ are respectively the classes of starlike and convex functions in $V$. The classes $V(\beta)$ and $U(\beta)$ have been studied by B. A. Uralegaddi, M. D. Ganigi and S. M. Sarangi [3]. They have shown that all functions in $V(\beta)$ are starlike and all functions in $U(\beta)$ are convex.

In this paper we investigate some properties of $f * g$ where $f, g \in V(\beta)$ or $U(\beta)$.

We need the following results [3].

Received June December, 1997.

1991 Mathematics Subject Classification. Primary 30C45.

Key words and phrases. univalent, starlike, convex, convolution. 
Theorem A. Let $f(z)=z+\sum_{n=2}^{\infty} a_{n} z^{n}$ be in $S$. If $\sum_{n=2}^{\infty}(n-\beta)\left|a_{n}\right| \leq \beta-1$ then $f \in M(\beta)$.

Theorem B. Let $f(z)=z+\sum_{n=2}^{\infty} a_{n} z^{n}$ be in $S$. If $\sum_{n=2}^{\infty} n(n-\beta)\left|a_{n}\right| \leq \beta-1$ then $f \in L(\beta)$..

Theorem C. A function $f(z)=z+\sum_{n=2}^{\infty} a_{n} z^{n}, a_{n} \geq 0$ is in $V(\beta)$ if and only if $\sum_{n=2}^{\infty}(n-\beta) a_{n} \leq \beta-1$.

Theorem D. A function $f(z)=z+\sum_{n=2}^{\infty} a_{n} z^{n}, a_{n} \geq 0$ is in $U(\beta)$ if and only if $\sum_{n=2}^{\infty} n(n-\beta) a_{n} \leq \beta-1$.

\section{Convolutions of functions in $V(\beta)$ and $U(\beta)$}

Theorem 1. If $f(z)=z+\sum_{n=2}^{\infty} a_{n} z^{n}, a_{n} \geq 0$ and $g(z)=z+\sum_{n=2}^{\infty} b_{n} z^{n}, b_{n} \geq 0$ are elements of $V(\beta)$, then $f(z) * g(z)=z+\sum_{n=2}^{\infty} a_{n} b_{n} z^{n}$ is an element of $V(\gamma)$, where $\gamma=\frac{6-8 \beta+3 \beta^{2}}{5-6 \beta+2 \beta^{2}}$.

Proof. Since $f(z)$ and $g(z) \in V(\beta)$, it follows that $\sum_{n=2}^{\infty} \frac{n-\beta}{\beta-1} a_{n} \leq 1$ and $\sum_{n=2}^{\infty} \frac{n-\beta}{\beta-1} b_{n}$ $\leq 1$. We want to show that $\sum_{n=2}^{\infty}(n-\gamma) a_{n} b_{n} \leq \gamma-1$. Equivalently we want to show

$$
\sum_{n=2}^{\infty} \frac{n-\beta}{\beta-1} a_{n} \leq 1
$$

and

$$
\sum_{n=2}^{\infty} \frac{n-\beta}{\beta-1} b_{n} \leq 1
$$

imply that

$$
\sum_{n=2}^{\infty} \frac{n-\gamma}{\gamma-1} a_{n} b_{n} \leq 1 \text { for all } \gamma=\gamma(\beta) \geq \frac{6-8 \beta+3 \beta^{2}}{5-6 \beta+2 \beta^{2}} .
$$

From (1) and (2) using Cauchy-Schawarz inequality, we get

$$
\sum_{n=2}^{\infty} \frac{n-\beta}{\beta-1} \sqrt{a_{n} b_{n}} \leq 1
$$

Hence it suffices to show that

From (4) it follows that

$$
\begin{gathered}
\frac{n-\gamma}{\gamma-1} a_{n} b_{n} \leq \frac{n-\beta}{\beta-1} \sqrt{a_{n} b_{n}}, \quad n=2,3, \ldots \\
\text { or } \sqrt{a_{n} b_{n}} \leq \frac{n-\beta}{\beta-1} \frac{\gamma-1}{n-\gamma} .
\end{gathered}
$$

$$
\sqrt{a_{n} b_{n}} \leq \frac{\beta-1}{n-\beta} \text { for each } n
$$


Therefore it is sufficient if we show that

$$
\frac{\beta-1}{n-\beta} \leq \frac{n-\beta}{\beta-1} \frac{\gamma-1}{n-\gamma} \text { for all } n
$$

The inequality (5) is equivalent to

$$
\gamma \geq \frac{1+n\left(\frac{\beta-1}{n-\beta}\right)^{2}}{1+\left(\frac{\beta-1}{n-\beta}\right)^{2}}
$$

The right-hand side of (6) is a decreasing function of $n, n=2,3, \ldots$. For $n=2$, we get

$$
\gamma \geq \frac{1+2\left(\frac{\beta-1}{2-\beta}\right)^{2}}{1+\left(\frac{\beta-1}{2-\beta}\right)^{2}}=\frac{6-8 \beta+3 \beta^{2}}{5-6 \beta+2 \beta^{2}} .
$$

The result follows.

Observe that $1<\gamma<4 / 3$.

The result is sharp, for the functins

$$
f(z)=g(z)=z+\left(\frac{\beta-1}{2-\beta}\right) z^{2}
$$

Remark 1. $V\left(\frac{6-8 \beta+3 \beta^{2}}{5-6 \beta+2 \beta^{2}}\right) \subset V^{*}\left(\frac{2-\beta^{2}}{3-2 \beta}\right)$ follows from the result [3]: If $f \in V(\beta)$ then $f \in V^{*}\left(\frac{4-3 \beta}{3-2 \beta}\right)$.

Remark 2. In the above theorem we have shown that if $f, g \in V(\beta)$ then $f * g \in$ $V\left(\frac{6-8 \beta+3 \beta^{2}}{5-6 \beta+2 \beta^{2}}\right)$. For given $h \in V\left(\frac{6-8 \beta+3 \beta^{2}}{5-6 \beta+2 \beta^{2}}\right)$ do there exist functions $f, g \in V(\beta)$ such that $h=f * g$ ? We shall show by an example that the answer is no. Let $f(z)=z+\sum_{n=2}^{\infty} a_{n} z^{n}$ and $g(z)=z+\sum_{n=2}^{\infty} b_{n} z^{n}$ be the functions in $V(\beta)$, then $a_{n} \leq \frac{\beta-1}{n-\beta}, b_{n} \leq \frac{\beta-1}{n-\beta}$. By the above theorem we have

$$
f * g=z+\sum_{n=2}^{\infty} a_{n} b_{n} z^{n} \in V\left(\frac{6-8 \beta+3 \beta^{2}}{5-6 \beta+2 \beta^{2}}\right) .
$$

Also note that $a_{n} b_{n} \leq\left(\frac{\beta-1}{n-\beta}\right)^{2}$.

Consider

$$
h(z)=z+\frac{\frac{6-8 \beta+3 \beta^{2}}{5-6 \beta+2 \beta^{2}}-1}{n-\frac{6-8 \beta+3 \beta^{2}}{5-6 \beta+2 \beta^{2}}} z^{n} \in V\left(\frac{6-8 \beta+3 \beta^{2}}{5-6 \beta+2 \beta^{2}}\right) .
$$

For this function we have

$$
\frac{\frac{6-8 \beta+3 \beta^{2}}{5-6 \beta+2 \beta^{2}}-1}{n-\frac{6-8 \beta+3 \beta^{2}}{5-6 \beta+2 \beta^{2}}}=\frac{(\beta-1)^{2}}{\left(5-6 \beta+2 \beta^{2}\right) n-\left(6-8 \beta+3 \beta^{2}\right)}>\frac{(\beta-1)^{2}}{(n-\beta)^{2}} \quad \text { for } n \geq 3 \text {. }
$$


i.e there is no $f$ and $g \in V(\beta)$ such that $f * g=h \in V\left(\frac{6-8 \beta+3 \beta^{2}}{5-6 \beta+2 \beta^{2}}\right)$.

Corollary. Let $f(z)$ and $g(z)$ be in $V(\beta)$. Then $h(z)=z+\sum_{n=2}^{\infty} \sqrt{a_{n} b_{n}} z^{n} \in V(\beta)$.

This result follows from the inequality (4). It is sharp for the same functions as in Theorem 1.

Theorem 2. If $f \in V(\beta)$ and $g \in V(\gamma)$ then $f * g \in V(\delta)$, where $\delta=\frac{6-4 \beta-4 \gamma+3 \beta \gamma}{5-3 \beta-3 \gamma+2 \beta \gamma}$.

Proof. Proceeding as in the proof of Theorem 1, we obtain

$$
\delta \geq \frac{1+\frac{n(\beta-1)(\gamma-1)}{(n-\beta)(n-\gamma)}}{1+\frac{(\beta-1)(\gamma-1)}{(n-\beta)(n-\gamma)}}
$$
The right-hand side of $(7)$ is a decreasing function of $n(n=2,3, \ldots)$. Taking $n=2$, we
get

$$
\delta \geq \frac{1+\frac{2(\beta-1)(\gamma-1)}{(2-\beta)(2-\gamma)}}{1+\frac{(\beta-1)(\gamma-1)}{(2-\beta)(2-\gamma)}}=\frac{6-4 \beta-4 \gamma+3 \beta \gamma}{5-3 \beta-3 \gamma+2 \beta \gamma}
$$

Corollary. If $f(z), g(z), h(z) \in V(\beta)$, then $f * g * h \in V\left(\frac{6-6 \beta+\beta^{3}}{7-9 \beta+3 \beta^{2}}\right)$.

Proof. From Theorem 1, we have $f * g \in V\left(\frac{6-8 \beta+3 \beta^{2}}{5-6 \beta+2 \beta^{2}}\right)$. Using Theorem 2, we get

$$
f * g * h \in V\left(\frac{6-4 \beta-4\left(\frac{6-8 \beta+3 \beta^{2}}{5-6 \beta+2 \beta^{2}}\right)+3 \beta\left(\frac{6-8 \beta+3 \beta^{2}}{5-6 \beta+2 \beta^{2}}\right)}{5-3 \beta-3\left(\frac{6-8 \beta+3 \beta^{2}}{5-6 \beta+2 \beta^{2}}\right)+2 \beta\left(\frac{6-8 \beta+3 \beta^{2}}{5-6 \beta+2 \beta^{2}}\right)}\right)
$$

i.e. $f * g * h \in V\left(\frac{6-6 \beta+\beta^{3}}{7-9 \beta+3 \beta^{2}}\right)$ :

For functions in the class $U(\beta)$ we have similar result. We shall prove:

Theorem 3. If $f \in U(\beta)$ and $g \in U(\gamma)$, then $f * g \in U(\delta)$, where $\delta=\frac{2(5-3 \beta-3 \gamma+2 \beta \gamma)}{9-5 \beta-5 \gamma+3 \beta \gamma}$.

Proof. From Theorem D, we have $\sum_{n=2}^{\infty} n(n-\beta) a_{n} \leq \beta-1$ and $\sum_{n=2}^{\infty} n(n-\gamma) b_{n} \leq$ $\gamma-1$. We wish to show that $\sum_{n=2}^{\infty} n(n-\delta) a_{n} b_{n} \leq \delta-1$. It is sufficient to show that

$$
\sum_{n=2}^{\infty} \frac{n(n-\beta)}{\beta-1} a_{n} \leq 1
$$

and

imply

$$
\sum_{n=2}^{\infty} \frac{n(n-\gamma)}{\gamma-1} b_{n} \leq 1
$$

$$
\sum_{n=2}^{\infty} \frac{n(n-\delta)}{\delta-1} a_{n} b_{n} \leq 1 \text { for all } \delta=\delta(\beta, \gamma) \geq \frac{2(5-3 \beta-3 \gamma+2 \beta \gamma)}{9-5 \beta-5 \gamma+3 \beta \gamma}
$$


Proceeding as in the proofs of Theorems 1 and 2, we get:

$$
\frac{n-\delta}{\delta-1} \leq \frac{n(n-\beta)(n-\gamma)}{(\beta-1)(\gamma-1)}
$$

or

$$
\delta \geq \frac{1+\frac{(\beta-1)(\gamma-1)}{(n-\beta)(n-\gamma)}}{1+\frac{(\beta-1)(\gamma-1)}{n(n-\beta)(n-\gamma)}}
$$

The right hand side of $(8)$ is a decreasing function of $n(n=2,3, \ldots)$. Taking $n=2$, we get

$$
\delta \geq \frac{2(5-3 \beta-3 \gamma+2 \beta \gamma)}{9-5 \beta-5 \gamma+3 \beta \gamma}
$$

The result follows.

Theorem 4. If $f \in V(\beta)$ and $g \in V(\gamma)$ then $f * g \in U(\delta)$, where $\delta=\frac{8-6 \beta-6 \gamma+5 \beta \gamma}{6-4 \beta-4 \gamma+3 \beta \gamma}$.

Proof. From Theorem C, we have $\sum_{n=2}^{\infty}(n-\beta) a_{n} \leq \beta-1$ and $\sum_{n=2}^{\infty}(n-\gamma) b_{n} \leq \gamma-1$. It follows that

$$
\sum_{n=2}^{\infty}(n-\beta)(n-\gamma) a_{n} b_{n} \leq(\beta-1)(\gamma-1) .
$$

We wish to show that $\sum_{n=2}^{\infty} n(n-\delta) a_{n} b_{n} \leq \delta-1$.

This is satisfied if

$$
\frac{n(n-\delta)}{\delta-1} \leq \frac{(n-\beta)(n-\gamma)}{(\beta-1)(\gamma-1)}
$$

i.e. for

$$
\delta \geq \frac{1+\frac{n^{2}(\beta-1)(\gamma-1)}{(n-\beta)(n-\gamma)}}{1+\frac{n(\beta-1)(\gamma-1)}{(n-\beta)(n-\gamma)}} .
$$

The right hand side of (9) is a decreasing function of $n(n=2,3, \ldots)$. Taking $n=2$, we get

$$
\delta \geq \frac{8-6 \beta-6 \gamma+5 \beta \gamma}{6-4 \beta-4 \gamma+3 \beta \gamma}
$$

The result follows.

The result is sharp for the functions

$$
f(z)=z+\frac{\beta-1}{2-\beta} z^{2} \in V(\beta) \text { and } g(z)=z+\frac{\gamma-1}{2-\gamma} z^{2} \in V(\gamma) .
$$

Putting $\beta=\gamma=4 / 3$ in Theorem 4, we get the following

Corollary. If $f, f \in V(4 / 3)$, then $f * g \in U(4 / 3)$.

Since $U(4 / 3) \subset V_{k}$ [3], the convolution of any two functions in $V(4 / 3)$ is convex. 
Theorem 5. If $f(z)=z+\sum_{n=2}^{\infty} a_{n} z^{n}, a_{n} \geq 0, \in V(\beta)$ and $g(z)=z+\sum_{n=2}^{\infty} b_{n} z^{n}$, with $\left|b_{i}\right| \leq 1, i=2,3, \ldots$, then $f * g \in M(\beta)$.

Proof. $\sum_{n=2}^{\infty}(n-\beta)\left|a_{n} b_{n}\right|=\sum_{n=2}^{\infty}(n-\beta) a_{n}\left|b_{n}\right| \leq \sum_{n=2}^{\infty}(n-\beta) a_{n} \leq \beta-1$.

Corollary. If $f(z) \in V(\beta)$ and $g(z)=z+\sum_{n=2}^{\infty} b_{n} z^{n}$, with $0 \leq b_{i} \leq 1, i=2,3, \ldots$, then $f * g \in V(\beta)$.

Theorem 6. let $f(z)=z+\sum_{n=2}^{\infty} a_{n} z^{n} \in U(\beta)$ and $g(z)=z+\sum_{n=2}^{\infty} b_{n} z^{n}$ with $\left|b_{i}\right| \leq 1, i=2,3 \ldots$, then $f * g \in L(\beta)$.

Proof.

$$
\begin{aligned}
& \sum_{n=2}^{\infty} n(n-\beta)\left|a_{n} b_{n}\right| \\
= & \sum_{n=2}^{\infty} n(n-\beta) a_{n}\left|b_{n}\right| \\
\leq & \sum_{n=2}^{\infty} n(n-\beta) a_{n} \leq \beta-1 .
\end{aligned}
$$
Corollary. If $f(z) \in U(\beta)$ and $g(z)=z+\sum_{n=2}^{\infty} b_{n} z^{n}$, with $0 \leq b_{i} \leq 1, i=2,3, \ldots$,
then $f * g \in U(\beta)$.

Theorem 7. If $f, g \in V(\beta)$, then $h(z)=z+\sum_{n=2}^{\infty}\left(a_{n}^{2}+b_{n}^{2}\right) z^{n} \in V(\delta)$ where $\delta=\frac{8-12 \beta+5 \beta^{2}}{6-8 \beta+3 \beta^{2}}$.

Proof. Since $\sum_{n=2}^{\infty}(n-\beta) a_{n} \leq \beta-1$, we have

$$
\sum_{n=2}^{\infty}\left(\frac{n-\beta}{\beta-1}\right)^{2} a_{n}^{2}<\left\{\sum_{n=2}^{\infty} \frac{n-\beta}{\beta-1} a_{n}\right\}^{2} \leq 1 .
$$

Simillarly

$$
\sum_{n=2}^{\infty}\left(\frac{n-\beta}{\beta-1}\right)^{2} b_{n}^{2} \leq 1
$$

From (10) and (11), we get

$$
\sum_{n=2}^{\infty} \frac{1}{2}\left(\frac{n-\beta}{\beta-1}\right)^{2}\left(a_{n}^{2}+b_{n}^{2}\right) \leq 1 .
$$

We want to find $\delta=\delta(\beta)$ such that

$$
\sum_{n=2}^{\infty}\left(\frac{n-\delta}{\delta-1}\right)\left(a_{n}^{2}+b_{n}^{2}\right) \leq 1
$$


Comparing this with (12), we see that (13) will be satisfied if

$$
\frac{n-\delta}{\delta-1} \leq \frac{1}{2}\left(\frac{n-\beta}{\beta-1}\right)^{2}
$$

or

$$
\delta \geq \frac{\left(\frac{n-\beta}{\beta-1}\right)^{2}+2 n}{\left(\frac{n-\beta}{\beta-1}\right)^{2}+2}
$$

Right-hand side of (14) is a decreasing function of $n(n=2,3, \ldots)$. Let $n=2$,

$$
\delta \geq \frac{8-12 \beta+5 \beta^{2}}{6-8 \beta+3 \beta^{2}} .
$$

The result is sharp for the function

$$
f(z)=g(z)=z+\frac{\beta-1}{2-\beta} z^{2} .
$$

Note that if in Theorem 4, we let $\gamma=\beta$, we get the same value for $\delta$ as here.

\section{References}

[1] St. Ruscheweyh and T. Sheil-Small, "Hadamard proudcts of schlicht functions and the Polya-Schoenberg conjecture," Comment. Math. Helv., 48(1973), 119-135.

[2] A. Schild and H. Silverman, "Convolutions of univalent functions with negative coefficients," Ann. Univ. M. Curie-sklodowska, Sect A, 29(1975), 99-106.

[3] B. A. Uralegaddi, M. D. Ganigi and S. M. Sarangi, "Univalent functions with positive coefficients," Tamkang J. Math, 25(1994), 225-230.

Department of Mathematics, Karnatak University, Dharwad-580 003, INDIA.

Department of Mathematics, S. D. M. College of Engg. \& Tech., Dharwad-580 002, INDIA. 